\title{
Global Dynamical Properties of Rational Higher-Order System of Difference Equations
}

\author{
A. Q. Khan iD and S. M. Qureshi \\ Department of Mathematics, University of Azad Jammu \& Kashmir, Muzaffarabad 13100, Pakistan \\ Correspondence should be addressed to A. Q. Khan; abdulqadeerkhan1@gmail.com
}

Received 17 April 2020; Accepted 5 June 2020; Published 15 July 2020

Academic Editor: Rigoberto Medina

Copyright (C) 2020 A. Q. Khan and S. M. Qureshi. This is an open access article distributed under the Creative Commons Attribution License, which permits unrestricted use, distribution, and reproduction in any medium, provided the original work is properly cited.

In this paper, global dynamical properties of rational higher-order system are explored in the interior of $\mathbb{R}_{+}^{3}$. It is explored that under certain parametric conditions, the discrete-time system has at most eight equilibria. By the method of linearization, local dynamics has been explored. It is explored that positive solution of the system is bounded, and moreover fixed point $P_{000}$ is globally stable if $\left(\alpha_{1} / \alpha_{2}\right)<1,\left(\alpha_{4} / \alpha_{5}\right)<1,\left(\alpha_{7} / \alpha_{8}\right)<1$. It is also investigated that the positive solution of the system under consideration converges to $P_{000}$. Lastly, theoretical results are confirmed by numerical simulation. The presented work is significantly extended and improves current results in the literature.

\section{Introduction}

It is a well-known fact that difference equations arise naturally as discrete analogues and as numerical solutions of differential as well as delay differential equations having applications in many fields like physics, biology, economy, and ecology. Recently, a lot of studies have been conducted concerning the global dynamics of difference equations and their systems [3-19]. It is really not easy to understand global dynamics of difference equations along their systems; particularly, investigating the global behavior of higher-order equations is a challenging job in recent years. Therefore, investigating the global dynamics of such difference equations along their systems is worth further consideration. For illustration, Gibbons et al. [20] explored global dynamics of the following equation:

$$
x_{n+1}=\frac{\alpha_{1}+\alpha_{2} x_{n-1}}{\alpha_{3}+x_{n}}
$$

where $\alpha_{\ell}(\ell=1,2,3)$ and $x_{-\ell}(\ell=1,0)$ are positive constants. Çinar [21] investigated the dynamics of the following equations:

$$
x_{n+1}=\frac{x_{n-1}}{1+\prod_{i=0}^{1} x_{n-i}}
$$

where $x_{-\ell}(\ell=1,0)$ are positive constants. Shojaei et al. [22] investigated global dynamics of the following difference equations:

$$
x_{n+1}=\frac{\alpha_{1} x_{n-2}}{\alpha_{2}+\alpha_{3} \prod_{i=0}^{2} x_{n-i}}
$$

where $\alpha_{\ell}(\ell=1,2,3)$ and $x_{-\ell}(\ell=2,1,0)$ are positive constants. Bajo and Liz [23] investigated the dynamics of the following difference equation:

$$
x_{n+1}=\frac{x_{n-1}}{\alpha_{1}+\alpha_{2} \prod_{i=0}^{1} x_{n-i}}
$$

where $\alpha_{\ell}(\ell=1,2)$ and $x_{-\ell}(\ell=1,0)$ are positive constants. Zhang et al. [24] have extended the work explored by numerous authors [21-23] to investigate the dynamics of the following rational system: 


$$
\begin{aligned}
& x_{n+1}=\frac{x_{n-2}}{\alpha_{2}+\prod_{i=0}^{2} y_{n-i}}, \\
& y_{n+1}=\frac{y_{n-1}}{\alpha_{1}+\prod_{i=0}^{2} x_{n-i}},
\end{aligned}
$$

where $\alpha_{\ell}(\ell=1,2)$ and $x_{\ell}, y_{-\ell}(\ell=2,1,0)$ are positive constants. Recently, Qureshi and Khan [25] investigated the global dynamics of the following rational system, which is extension of the work [21-24]:

$$
\begin{aligned}
& x_{n+1}=\frac{\alpha_{1} x_{n-1}}{\alpha_{2}+\alpha_{3} \prod_{i=0}^{1} y_{n-i}}, \\
& y_{n+1}=\frac{\alpha_{4} y_{n-1}}{\alpha_{5}+\alpha_{6} \prod_{i=0}^{1} z_{n-i}}, \\
& z_{n+1}=\frac{\alpha_{7} z_{n-1}}{\alpha_{8}+\alpha_{9} \prod_{i=0}^{1} x_{n-i}},
\end{aligned}
$$

where $\alpha_{\ell}(\ell=1,2, \ldots, 9)$ and $x_{-\ell}, y_{-\ell}, z_{-\ell}(\ell=1,0)$ are positive constants. Inspired from aforesaid studies, we will extend the work studied by numerous authors [21-25] to investigate global dynamics of the following $3 k+3$-dimensional system:

$$
\begin{aligned}
& x_{n+1}=\frac{\alpha_{1} x_{n-k}}{\alpha_{2}+\alpha_{3} \prod_{i=0}^{k} y_{n-i}} \\
& y_{n+1}=\frac{\alpha_{4} y_{n-k}}{\alpha_{5}+\alpha_{6} \prod_{i=0}^{k} z_{n-i}}, \\
& z_{n+1}=\frac{\alpha_{7} z_{n-k}}{\alpha_{8}+\alpha_{9} \prod_{i=0}^{k} x_{n-i}},
\end{aligned}
$$

where $\alpha_{\ell}(\ell=1,2, \ldots, 9)$ and $x_{-\ell}, \ell_{-\ell}, z_{-\ell}(\ell=k,-k+$ $1, \ldots, 1,0)$ are positive constants.

The organization of this paper is as follows. In Section 2, existence of equilibria in $\mathbb{R}_{+}^{3}$ and corresponding linearized form are investigated. Section 3 deals with the study of local dynamics about equilibrium points. Boundedness of positive solution for the discrete-time system is studied in Section 4. Further, global dynamics about $P_{000}$ is explored in Section 5. In Section 6, we studied the rate of convergence which converges to $P_{000}$ of the system. Theoretical results are numerically verified in Section 7, while concluding remarks are given in Section 8.

\section{Equilibria and Linearized Form of System (7)}

The existence of equilibrium solution in the interior of $\mathbb{R}_{+}^{3}$ and linearized form about $\Lambda=(x, y, z)$ of system (7) are investigated in this section. So, existence of equilibrium solution can be summarized as the following lemma.

Lemma 1. In the interior of $\mathbb{R}_{+}^{3}$, discrete-time system (7) has at most eight equilibria. More precisely, (i) $\forall \alpha_{\ell}(\ell=1,2, \ldots, 9), \quad P_{000}(0,0,0)$ is the unique boundary point of discrete-time system (7).

(ii) $P_{x 0 z}\left(\sqrt{[k+1]}\left(\alpha_{7}-\alpha_{8} / \alpha_{9}\right), 0, \sqrt{[k+1]}\left(\alpha_{4}-\right.\right.$ $\left.\left.\alpha_{5} / \alpha_{6}\right)\right)$ is the boundary equilibrium point of system (7) if $\alpha_{1}=\alpha_{2}, \alpha_{4}>\alpha_{5}$ and $\alpha_{7}>\alpha_{8}$.

(iii) $P_{x y 0}\left(\sqrt{[k+1]}\left(\alpha_{7}-\alpha_{8} / \alpha_{9}\right), \sqrt{[k+1]}\left(\alpha_{1}-\right.\right.$ $\left.\left.\alpha_{2} / \alpha_{3}\right), 0\right)$ is the boundary equilibrium point of system (7) if $\alpha_{1}>\alpha_{2}, \alpha_{4}=\alpha_{5}$ and $\alpha_{7}>\alpha_{8}$.

(iv) $P_{0 y z}\left(0, \sqrt{[k+1]}\left(\alpha_{1}-\alpha_{2} / \alpha_{3}\right), \sqrt{[k+1]}\left(\alpha_{4}-\right.\right.$ $\left.\alpha_{5} / \alpha_{6}\right)$ ) is the boundary equilibrium point of system (7) if $\alpha_{1}>\alpha_{2}, \alpha_{4}>\alpha_{5}$ and $\alpha_{7}=\alpha_{8}$.

(v) $P_{x 00}\left(\sqrt{[k+1]}\left(\alpha_{7}-\alpha_{8} / \alpha_{9}\right), 0,0\right)$ is the boundary equilibrium point of discrete-time system (7) if $\alpha_{1}=\alpha_{2}, \alpha_{4}=\alpha_{5}$ and $\alpha_{7}>\alpha_{8}$.

(vi) $P_{00 z}\left(0,0, \sqrt{[k+1]}\left(\alpha_{4}-\alpha_{5} / \alpha_{6}\right)\right)$ is the boundary equilibrium point of system (7) if $\alpha_{1}=\alpha_{2}$, $\alpha_{4}>\alpha_{5}$ and $\alpha_{7}=\alpha_{8}$.

(vii) $P_{0 y 0}\left(0, \sqrt{[k+1]}\left(\alpha_{1}-\alpha_{2} / \alpha_{3}\right), 0\right)$ is the boundary equilibrium point of discrete-time system (7) if $\alpha_{1}>\alpha_{2}, \alpha_{4}=\alpha_{5}$ and $\alpha_{7}=\alpha_{8}$.

(viii) $P_{x y z}\left(\sqrt{[k+1]}\left(\alpha_{7}-\alpha_{8} / \alpha_{9}\right), \sqrt{[k+1]}\left(\alpha_{1}-\right.\right.$ $\left.\left.\alpha_{2} / \alpha_{3}\right), \sqrt{[k+1]}\left(\alpha_{4}-\alpha_{5} / \alpha_{6}\right)\right)$ is the unique positive equilibrium point of system (7) if $\alpha_{1}>\alpha_{2}$, $\alpha_{4}>\alpha_{5}$ and $\alpha_{7}>\alpha_{8}$.

Hereafter, we establish the corresponding linearized form of (7). For this, one has the following map in order to construct the corresponding linearized form:

$$
\begin{aligned}
& \left(\beta, \beta_{n}, \ldots, \beta_{n-k}, \gamma, \gamma_{n}, \ldots, \gamma_{n-k}, \delta, \delta_{n}, \ldots, \delta_{n-k}\right) \\
& \quad \mapsto\left(x_{n+1}, x_{n}, \ldots, x_{n-k+1}, y_{n+1}, y_{n}, \ldots, y_{n-k+1}, z_{n+1}, z_{n}, \ldots, z_{n-k+1}\right),
\end{aligned}
$$

where

$$
\begin{gathered}
\beta=\frac{\alpha_{1} x_{n-k}}{\alpha_{2}+\alpha_{3} \prod_{i=0}^{k} y_{n-i}}, \\
\beta_{n}=x_{n}, \ldots, \beta_{n-k}=x_{n-k+1}, \\
\gamma=\frac{\alpha_{4} y_{n-k}}{\alpha_{5}+\alpha_{6} \prod_{i=0}^{k} z_{n-i}}, \\
\gamma_{n}=y_{n}, \ldots, \gamma_{n-k}=y_{n-k+1}, \\
\delta=\frac{\alpha_{7} z_{n-k}}{\alpha_{8}+\alpha_{9} \prod_{i=0}^{k} x_{n-i}}, \\
\delta_{n}=z_{n}, \ldots, \delta_{n-k}=z_{n-k+1} .
\end{gathered}
$$

Finally, $\left.J\right|_{\wedge}$ about $\wedge$ under map (8) becomes 
Discrete Dynamics in Nature and Society

3

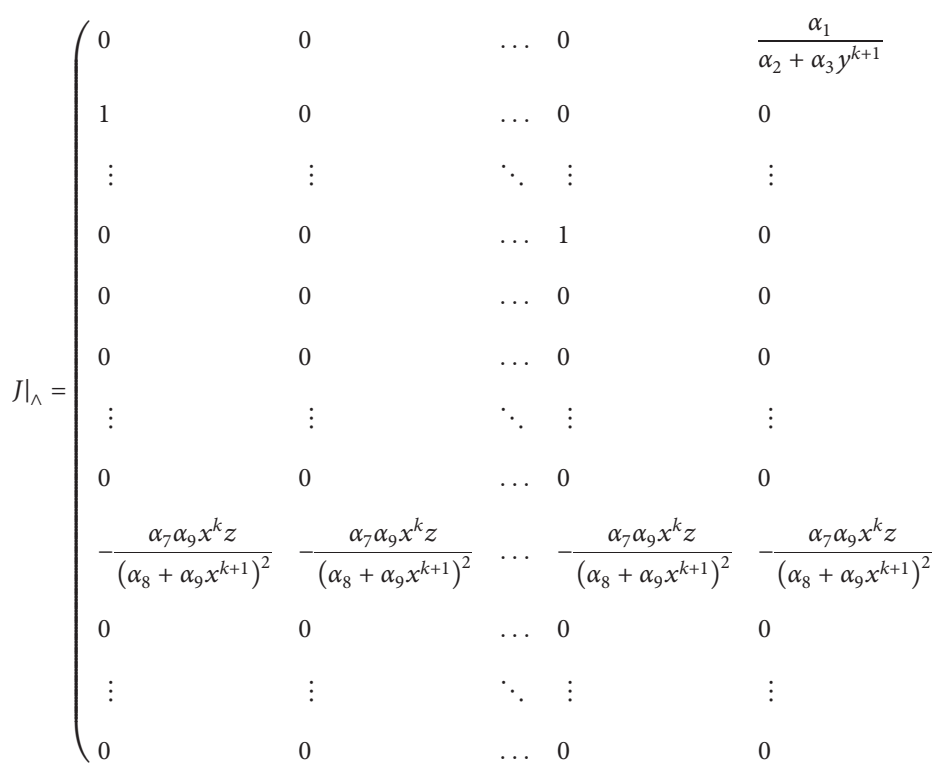

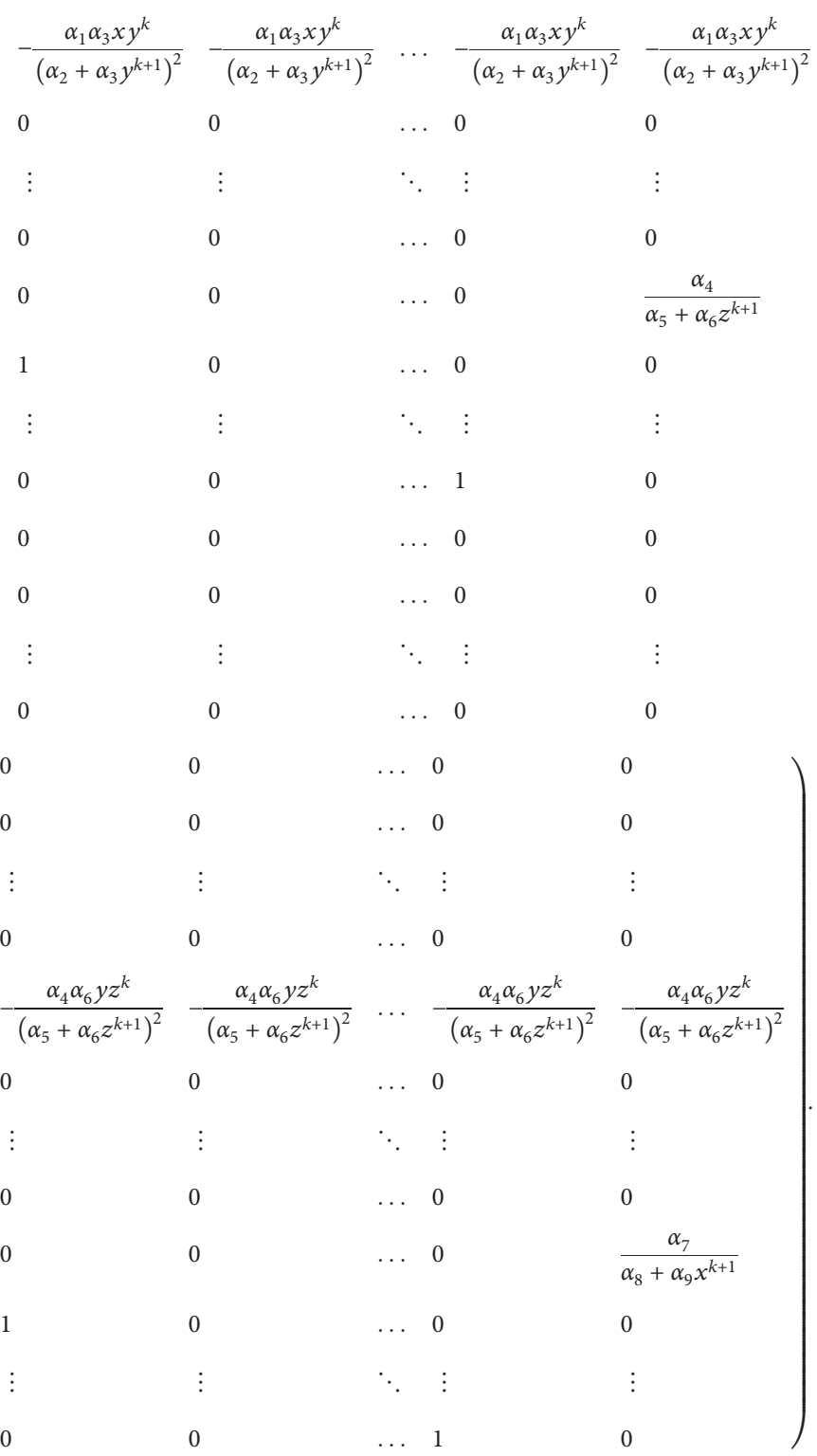

(10) 


\section{Local Dynamics about Equilibria}

By Theorem 1.5 of [1], the detailed local stability analysis about boundary equilibria $P_{000}, P_{x 0 z}, P_{x y 0}, P_{0 y z}, P_{x 00}, P_{00 z}$, and $P_{0 y 0}$ and the positive equilibrium point $P_{x y z}$ will be investigated in this section.

\subsection{Local Dynamics about Boundary Points}

Theorem 1. $P_{000}$ of system (7) is a sink if

$$
\frac{\alpha_{1}}{\alpha_{2}}<1, \frac{\alpha_{4}}{\alpha_{5}}<1, \frac{\alpha_{7}}{\alpha_{8}}<1
$$

Proof. About the equilibrium point $P_{000}$, (10) takes the following form:

$$
\left.J\right|_{P_{000}}=\left(\begin{array}{ccccccccccccccc}
0 & 0 & \ldots & 0 & \frac{\alpha_{1}}{\alpha_{2}} & 0 & 0 & \ldots & 0 & 0 & 0 & 0 & \ldots & 0 & 0 \\
1 & 0 & \ldots & 0 & 0 & 0 & 0 & \ldots & 0 & 0 & 0 & 0 & \ldots & 0 & 0 \\
\vdots & \vdots & \ddots & \vdots & \vdots & \vdots & \vdots & \ddots & \vdots & \vdots & \vdots & \vdots & \ddots & \vdots & \vdots \\
0 & 0 & \ldots & 1 & 0 & 0 & 0 & \ldots & 0 & 0 & 0 & 0 & \ldots & 0 & 0 \\
0 & 0 & \ldots & 0 & 0 & 0 & 0 & \ldots & 0 & \frac{\alpha_{4}}{\alpha_{5}} & 0 & 0 & \ldots & 0 & 0 \\
0 & 0 & \ldots & 0 & 0 & 1 & 0 & \ldots & 0 & 0 & 0 & 0 & \ldots & 0 & 0 \\
\vdots & \vdots & \ddots & \vdots & \vdots & \vdots & \vdots & \ddots & \vdots & \vdots & \vdots & \vdots & \ddots & \vdots & \vdots \\
0 & 0 & \ldots & 0 & 0 & 0 & 0 & \ldots & 1 & 0 & 0 & 0 & \ldots & 0 & 0 \\
0 & 0 & \ldots & 0 & 0 & 0 & 0 & \ldots & 0 & 0 & 0 & 0 & \ldots & 0 & \frac{\alpha_{7}}{\alpha_{8}} \\
0 & 0 & \ldots & 0 & 0 & 0 & 0 & \ldots & 0 & 0 & 1 & 0 & \ldots & 0 & 0 \\
\vdots & \vdots & \ddots & \vdots & \vdots & \vdots & \vdots & \ddots & \vdots & \vdots & \vdots & \vdots & \ddots & \vdots & \vdots \\
0 & 0 & \ldots & 0 & 0 & 0 & 0 & \ldots & 0 & 0 & 0 & 0 & \ldots & 1 & 0
\end{array}\right)
$$

Now, if $v_{1}, v_{2}, \ldots, v_{k+1}, \ldots, v_{2 k+1}, \ldots, v_{3 k+3}$ denotes characteristic roots of $P_{000}$, the diagonal matrix $\Omega=\operatorname{diag}\left(\rho_{1}, \rho_{2}, \ldots, \rho_{k+1}, \ldots, \rho_{2 k+1}, \ldots, \rho_{3 k+3}\right)$, where

$$
\begin{aligned}
& \rho_{1}=\rho_{k+2}=\rho_{2 k+3}=1, \\
& \begin{aligned}
& \rho_{1+\varsigma}=\rho_{k+2+\varsigma}= \rho_{2 k+3+\varsigma}=1-\varsigma \varepsilon \\
& 1 \leq \varsigma \leq k \\
& 0<\varepsilon<1, \\
& 0<\varepsilon<\min \left\{\frac{1}{k}\left(1-\frac{\alpha_{1}}{\alpha_{2}}\right), \frac{1}{k}\left(1-\frac{\alpha_{4}}{\alpha_{5}}\right), \frac{1}{k}\left(1-\frac{\alpha_{7}}{\alpha_{8}}\right)\right\} .
\end{aligned}
\end{aligned}
$$

Now,

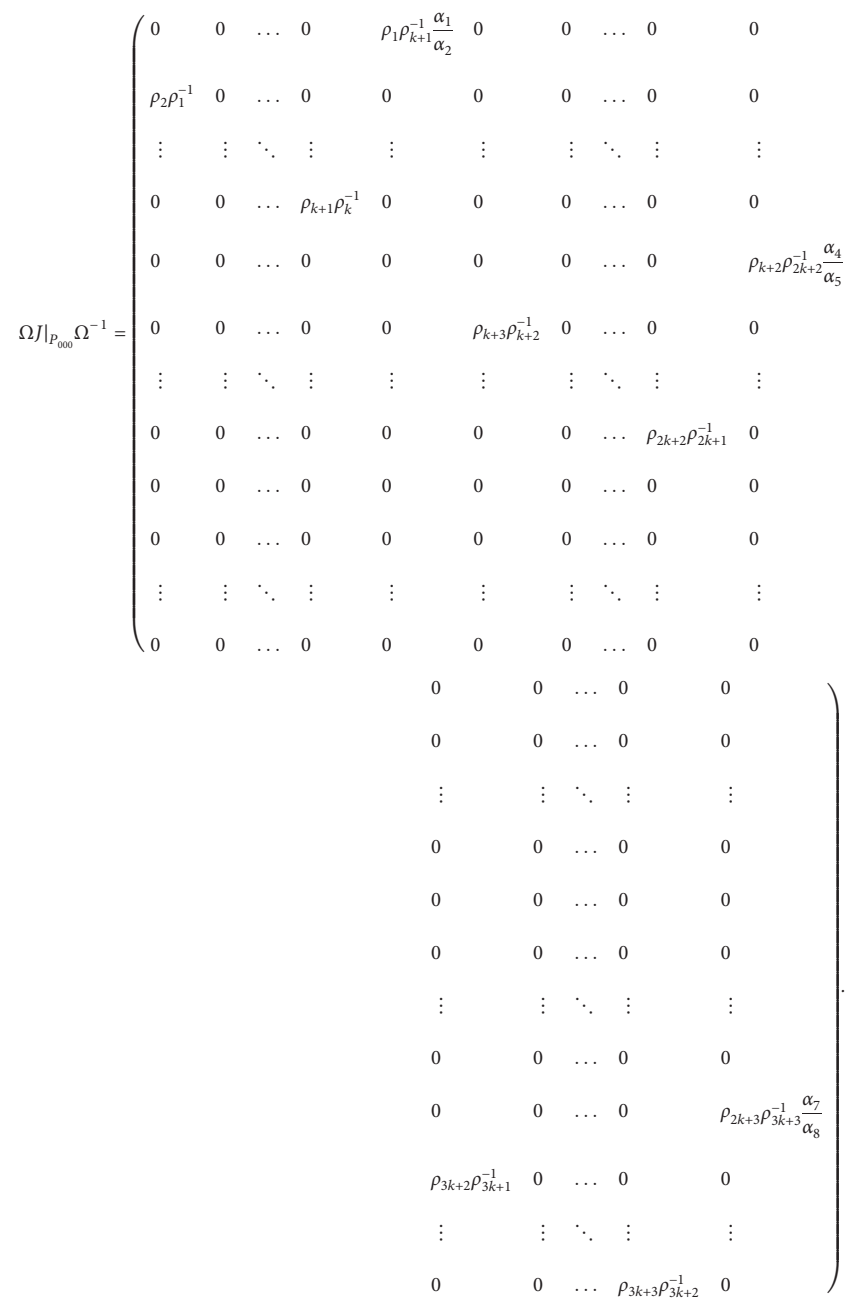

So,

$$
\begin{aligned}
& 0<\rho_{k+1}<\cdots<\rho_{2}<\rho_{1}, \\
& 0<\rho_{2 k+2}<\cdots<\rho_{k+2}, \\
& 0<\rho_{3 k+3}<\cdots<\rho_{2 k+3} .
\end{aligned}
$$

From (16), one obtains

$$
\begin{gathered}
\rho_{2} \rho_{1}^{-1}<1, \ldots, \rho_{k+1} \rho_{k}^{-1}<1, \\
\rho_{k+3} \rho_{k+2}^{-1}<1, \ldots, \rho_{2 k+2} \rho_{2 k+1}^{-1}<1, \\
\rho_{2 k+4} \rho_{2 k+3}^{-1}<1, \ldots, \rho_{3 k+3} \rho_{3 k+2}^{-1}<1 .
\end{gathered}
$$

From (13) and (14), one obtains

$$
\rho_{1} \rho_{k+1}^{-1} \frac{\alpha_{1}}{\alpha_{2}}=\frac{\alpha_{1}}{\alpha_{2}} \frac{1}{1-k \varepsilon}<1
$$

$$
\begin{gathered}
\rho_{k+2} \rho_{2 k+2}^{-1} \frac{\alpha_{4}}{\alpha_{5}}=\frac{\alpha_{4}}{\alpha_{5}} \frac{1}{1-k \varepsilon}<1, \\
\rho_{2 k+3} \rho_{3 k+3}^{-1} \frac{\alpha_{7}}{\alpha_{8}}=\frac{\alpha_{7}}{\alpha_{8}} \frac{1}{1-k \varepsilon}<1 .
\end{gathered}
$$

In view of (17) and (18), one obtains 


$$
\begin{aligned}
\max _{1 \leq m \leq 3 k+3}\left|\rho_{m}\right| & =|\Omega J|_{P_{000}} \Omega^{-1} \mid \\
& =\max \left\{\rho_{1} \rho_{k+1}^{-1} \frac{\alpha_{1}}{\alpha_{2}}, \rho_{2} \rho_{1}^{-1}, \ldots, \rho_{k+1} \rho_{k}^{-1}, \rho_{k+3} \rho_{k+2}^{-1}, \ldots, \rho_{2 k+2} \rho_{2 k+1}^{-1}, \rho_{3 k+2} \rho_{3 k+1}^{-1}, \ldots, \rho_{3 k+3} \rho_{3 k+2}^{-1}, \rho_{k+2} \rho_{2 k+2}^{-1} \frac{\alpha_{4}}{\alpha_{5}}, \rho_{2 k+3} \rho_{3 k+3}^{-1} \frac{\alpha_{7}}{\alpha_{8}}\right\}<1 .
\end{aligned}
$$

From (19), one gets the required statement.

Theorem 2. $P_{x 0 z}$ of (7) is unstable.

Proof. About the equilibrium point $P_{x 0 z}$, (10) takes the following form:

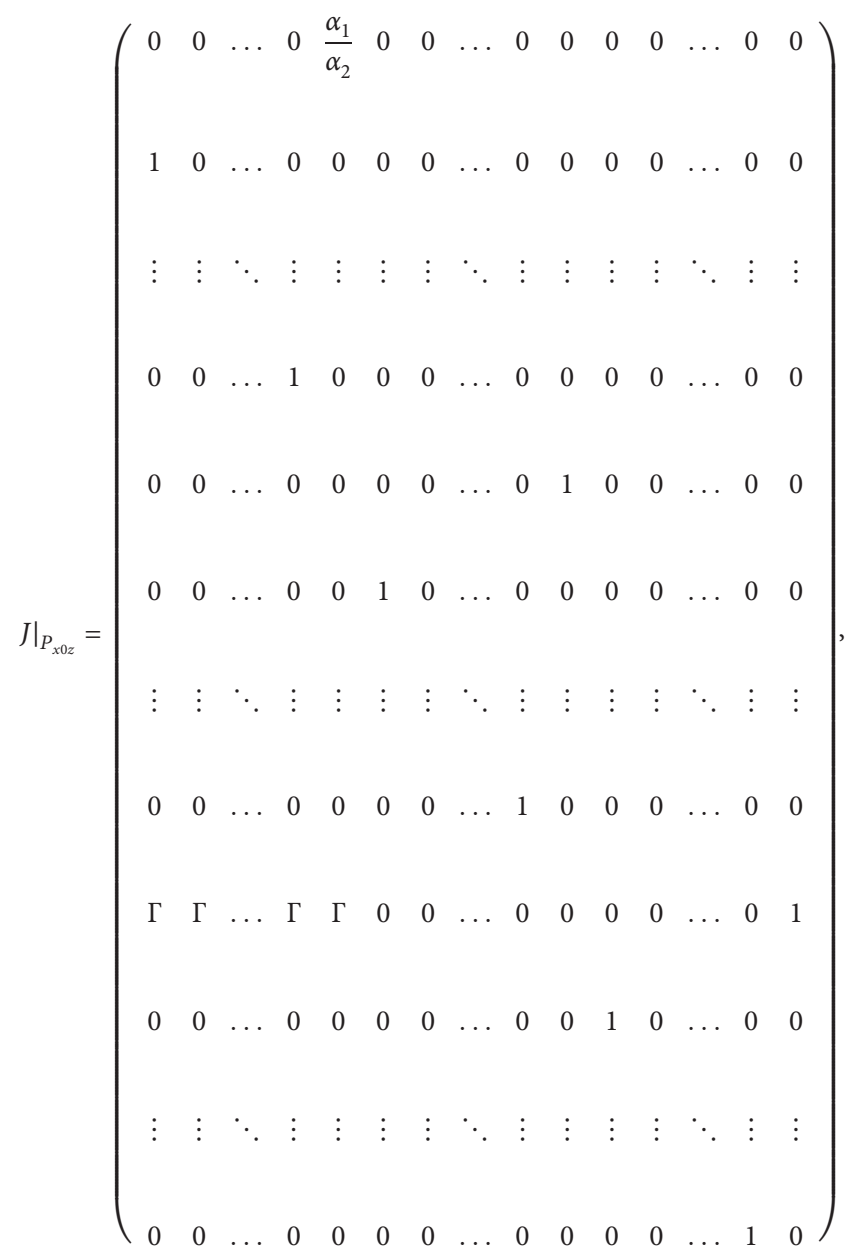

where

$$
\Gamma=-\frac{\alpha_{9}}{\alpha_{7}} \sqrt{[k+1]} \frac{\alpha_{4}-\alpha_{5}}{\alpha_{6}} \sqrt{\left[\frac{k}{k+1}\right]} \frac{\alpha_{7}-\alpha_{8}}{\alpha_{9}} .
$$

Now, if $v_{1}, v_{2}, \ldots, v_{k+1}, \ldots, v_{2 k+1}, \ldots, v_{3 k+3}$ denotes characteristic roots of $P_{x 0 z}$, the diagonal matrix $\Omega=\operatorname{diag}\left(\rho_{1}, \rho_{2}, \ldots, \rho_{k+1}, \ldots, \rho_{2 k+1}, \ldots, \rho_{3 k+3}\right)$, where (13) holds. Moreover,

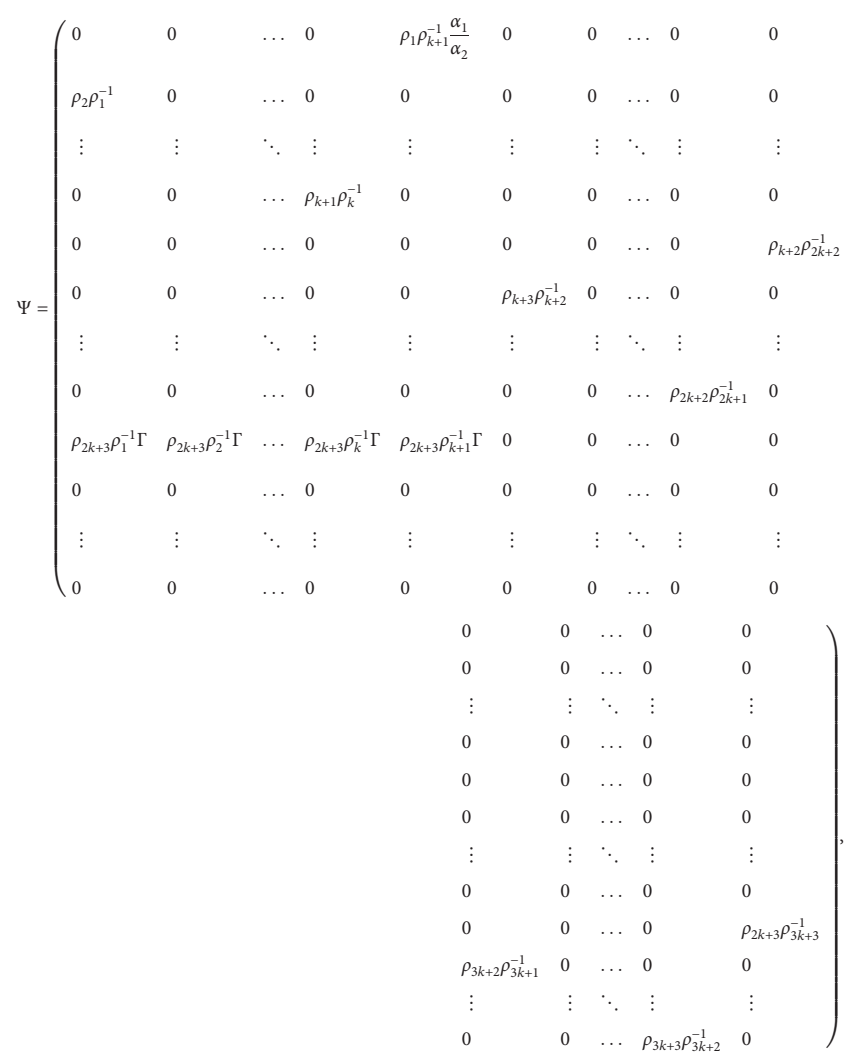

where $\Phi=\left.\Omega J\right|_{P_{x 0 z}} \Omega^{-1}$. From (16) and (17), we obtain

$$
\rho_{1} \rho_{k+1}^{-1} \frac{\alpha_{1}}{\alpha_{2}}=\frac{\alpha_{1}}{\alpha_{2}} \frac{1}{1-k \varepsilon}<1 .
$$

From (23), one gets $\varepsilon<1-\left(\alpha_{1} / \alpha_{2}\right)$. But $\rho_{k+2} \rho_{2 k+2}^{-1}=\rho_{2 k+2}^{-1}=(1 / 1-k \varepsilon)>1$, and hence $P_{x 0 z}$ of $(7)$ is unstable.

Similarly, local dynamics about $P_{x y 0}$ and $P_{0 y z}$ of system under consideration can be summarized as follows.

Theorem 3. (i) $P_{x y 0}$ of system (7) is unstable.

(ii) $P_{0 y z}$ of system (7) is unstable.

Proof. Same as proof of Theorems 1 and 2.

Theorem 4. $P_{x 00}$ of system (7) is unstable.

Proof. About the equilibrium point $P_{x 00}$, (10) takes the following form: 
$\left.J\right|_{P_{x 00}}=\left(\begin{array}{ccccccccccccccc}0 & 0 & \ldots & 0 & \frac{\alpha_{1}}{\alpha_{2}} & 0 & 0 & \ldots & 0 & 0 & 0 & 0 & \ldots & 0 & 0 \\ 1 & 0 & \ldots & 0 & 0 & 0 & 0 & \ldots & 0 & 0 & 0 & 0 & \ldots & 0 & 0 \\ \vdots & \vdots & \ddots & \vdots & \vdots & \vdots & \vdots & \ddots & \vdots & \vdots & \vdots & \vdots & \ddots & \vdots & \vdots \\ 0 & 0 & \ldots & 1 & 0 & 0 & 0 & \ldots & 0 & 0 & 0 & 0 & \ldots & 0 & 0 \\ 0 & 0 & \ldots & 0 & 0 & 0 & 0 & \ldots & 0 & \frac{\alpha_{4}}{\alpha_{5}} & 0 & 0 & \ldots & 0 & 0 \\ 0 & 0 & \ldots & 0 & 0 & 1 & 0 & \ldots & 0 & 0 & 0 & 0 & \ldots & 0 & 0 \\ \vdots & \vdots & \ddots & \vdots & \vdots & \vdots & \vdots & \ddots & \vdots & \vdots & \vdots & \vdots & \ddots & \vdots & \vdots \\ 0 & 0 & \ldots & 0 & 0 & 0 & 0 & \ldots & 1 & 0 & 0 & 0 & \ldots & 0 & 0 \\ 0 & 0 & \ldots & 0 & 0 & 0 & 0 & \ldots & 0 & 0 & 0 & 0 & \ldots & 0 & 1 \\ 0 & 0 & \ldots & 0 & 0 & 0 & 0 & \ldots & 0 & 0 & 1 & 0 & \ldots & 0 & 0 \\ \vdots & \vdots & \ddots & \vdots & \vdots & \vdots & \vdots & \ddots & \vdots & \vdots & \vdots & \vdots & \ddots & \vdots & \vdots \\ 0 & 0 & \ldots & 0 & 0 & 0 & 0 & \ldots & 0 & 0 & 0 & 0 & \ldots & 1 & 0\end{array}\right)$.
Now, if $v_{1}, v_{2}, \ldots, v_{k+1}, \ldots, v_{2 k+1}, \ldots, v_{3 k+3}$ denotes characteristic roots of $P_{x 00}$, the diagonal matrix $\Omega=\operatorname{diag}\left(\rho_{1}, \rho_{2}, \ldots, \rho_{k+1}, \ldots, \rho_{2 k+1}, \ldots, \rho_{3 k+3}\right)$, where (13) holds. Moreover,

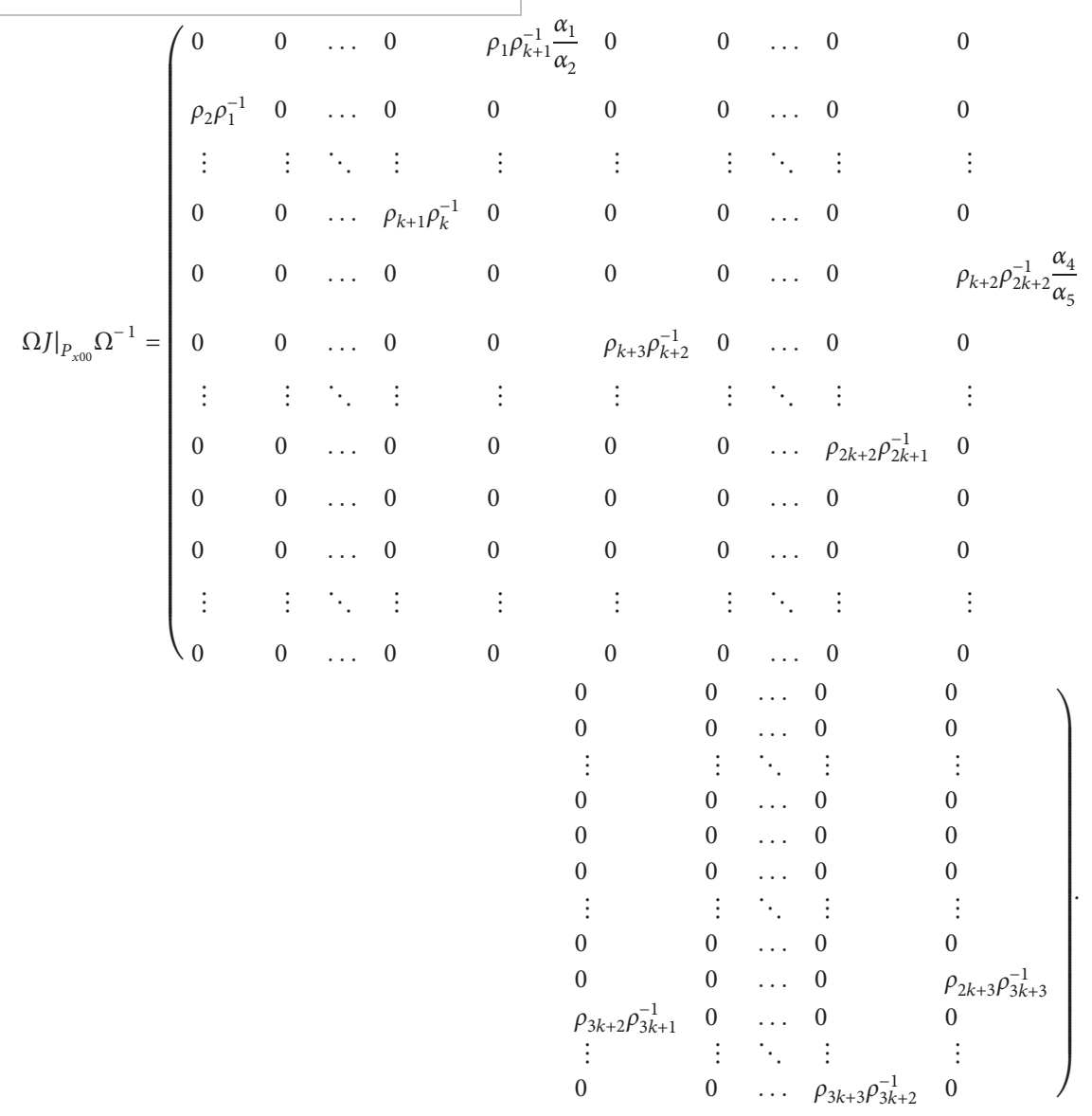

From (16) and (17), we obtain

$$
\rho_{2 k+3} \rho_{3 k+3}^{-1}=\rho_{3 k+3}^{-1}=\frac{1}{1-k \varepsilon}>1
$$

which gives $P_{x 00}$ of (7) is unstable.

Similarly, local dynamics about $P_{00 z}, P_{0 y 0}$ of system under consideration can be summarized as follows. 
Theorem 5. (i) $P_{00 z}$ of system (7) is unstable.

(ii) $P_{0 y 0}$ of system (7) is unstable.

Proof. Same as proof of Theorems 1, 2, and 4 .

\subsection{Local Dynamics about Positive Point}

Theorem 6. $P_{x y z}$ of (7) is unstable.

Proof. About the equilibrium point $P_{x y z}$, (10) takes the following form:

$$
\left.J\right|_{P_{x y z}}=\left(\begin{array}{ccccccccccccccc}
0 & 0 & \ldots & 0 & 1 & \Lambda_{1} & \Lambda_{1} & \ldots & \Lambda_{1} & \Lambda_{1} & 0 & 0 & \ldots & 0 & 0 \\
1 & 0 & \ldots & 0 & 0 & 0 & 0 & \ldots & 0 & 0 & 0 & 0 & \ldots & 0 & 0 \\
\vdots & \vdots & \ddots & \vdots & \vdots & \vdots & \vdots & \ddots & \vdots & \vdots & \vdots & \vdots & \ddots & \vdots & \vdots \\
0 & 0 & \ldots & 1 & 0 & 0 & 0 & \ldots & 0 & 0 & 0 & 0 & \ldots & 0 & 0 \\
0 & 0 & \ldots & 0 & 0 & 0 & 0 & \ldots & 0 & 1 & \Lambda_{2} & \Lambda_{2} & \ldots & \Lambda_{2} & \Lambda_{2} \\
0 & 0 & \ldots & 0 & 0 & 1 & 0 & \ldots & 0 & 0 & 0 & 0 & \ldots & 0 & 0 \\
\vdots & \vdots & \ddots & \vdots & \vdots & \vdots & \vdots & \ddots & \vdots & \vdots & \vdots & \vdots & \ddots & \vdots & \vdots \\
0 & 0 & \ldots & 0 & 0 & 0 & 0 & \ldots & 1 & 0 & 0 & 0 & \ldots & 0 & 0 \\
\Gamma & \Gamma & \ldots & \Gamma & \Gamma & 0 & 0 & \ldots & 0 & 0 & 0 & 0 & \ldots & 0 & 1 \\
0 & 0 & \ldots & 0 & 0 & 0 & 0 & \ldots & 0 & 0 & 1 & 0 & \ldots & 0 & 0 \\
\vdots & \vdots & \ddots & \vdots & \vdots & \vdots & \vdots & \ddots & \vdots & \vdots & \vdots & \vdots & \ddots & \vdots & \vdots \\
0 & 0 & \ldots & 0 & 0 & 0 & 0 & \ldots & 0 & 0 & 0 & 0 & \ldots & 1 & 0
\end{array}\right) .
$$

where

$$
\begin{aligned}
& \Lambda_{1}=-\frac{\alpha_{3}}{\alpha_{1}} \sqrt{\left[\frac{k}{k+1}\right]} \frac{\alpha_{1}-\alpha_{2}}{\alpha_{3}} \sqrt{[k+1]} \frac{\alpha_{7}-\alpha_{8}}{\alpha_{9}}, \\
& \Lambda_{2}=-\frac{\alpha_{6}}{\alpha_{4}} \sqrt{[k+1]} \frac{\alpha_{1}-\alpha_{2}}{\alpha_{3}} \sqrt{\left[\frac{k}{k+1}\right]} \frac{\alpha_{4}-\alpha_{5}}{\alpha_{6}},
\end{aligned}
$$

and $\Gamma$ is depicted in (21). Moreover,

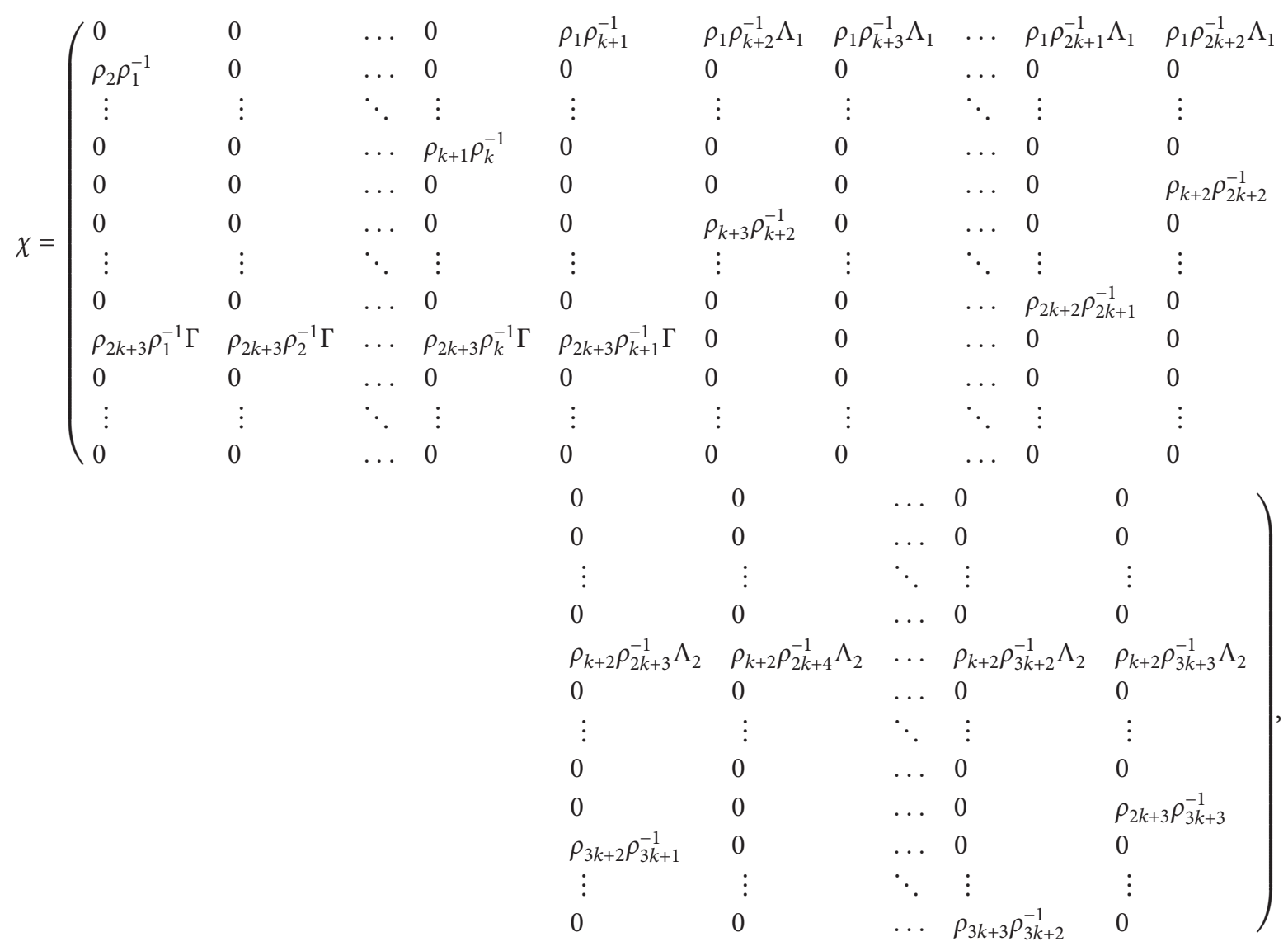


where $\chi=\left.\Omega J\right|_{P_{x y z}} \Omega^{-1}$. From (16) and (17), we obtain

$$
\begin{aligned}
\rho_{1} \rho_{k+1}^{-1}+\rho_{1}\left(\rho_{k+2}^{-1}+\rho_{k+3}^{-1}+\cdots+\rho_{2 k+1}^{-1}+\rho_{2 k+2}^{-1}\right) \Lambda_{1} & =\frac{1}{1-k \varepsilon}+\left(1+\frac{1}{1-\varepsilon}+\cdots+\frac{1}{1-(k-1) \varepsilon}+\frac{1}{1-k \varepsilon}\right) \Lambda_{1}>1 \\
\rho_{k+2} \rho_{2 k+2}^{-1}+\rho_{k+2}\left(\rho_{2 k+3}^{-1}+\rho_{2 k+4}^{-1}+\cdots \rho_{3 k+2}^{-1}+\rho_{3 k+3}^{-1}\right) \Lambda_{2} & =\frac{1}{1-k \varepsilon}+\left(1+\frac{1}{1-\varepsilon}+\cdots+\frac{1}{1-(k-1) \varepsilon}+\frac{1}{1-k \varepsilon}\right) \Lambda_{2}>1 \\
\rho_{2 k+3} \rho_{3 k+3}^{-1}+\rho_{2 k+3}\left(\rho_{1}^{-1}+\rho_{2}^{-1}+\cdots+\rho_{k}^{-1}+\rho_{k+1}^{-1}\right) \Gamma & =\frac{1}{1-k \varepsilon}+\left(1+\frac{1}{1-\varepsilon}+\cdots+\frac{1}{1-(k-1) \varepsilon}+\frac{1}{1-k \varepsilon}\right) \Lambda_{2}>1
\end{aligned}
$$

From (30), one can conclude that $P_{x y z}$ of (7) is unstable.

\section{Boundedness}

The boundedness of positive solution of (7) is investigated in this section, as follows.

Theorem 7. If $\left\{\Omega_{n}\right\}_{n=-k}^{\infty}=\left\{\left(x_{n}, y_{n}, z_{n}\right)\right\}_{n=-k}^{\infty}$ is a positive solution of (7), then following holds for $v \geq 0$ :

$$
\begin{aligned}
& 0<x_{n} \leq \begin{cases}\left(\frac{\alpha_{1}}{\alpha_{2}}\right)^{v+1} x_{-k}, & \text { if } n=(k+1) v+1, \\
\left(\frac{\alpha_{1}}{\alpha_{2}}\right)^{v+1} x_{-k+1}, & \text { if } n=(k+1) v+2, \\
\vdots & \\
\left(\frac{\alpha_{1}}{\alpha_{2}}\right)^{v+1} x_{-1}, & \text { if } n=(k+1) v+k, \\
\left(\frac{\alpha_{1}}{\alpha_{2}}\right)^{v+1} x_{0}, & \text { if } n=(k+1) v+(k+1),\end{cases} \\
& 0<y_{n} \leq \begin{cases}\left(\frac{\alpha_{4}}{\alpha_{5}}\right)^{v+1} y_{-k}, & \text { if } n=(k+1) v+1, \\
\left(\frac{\alpha_{4}}{\alpha_{5}}\right)^{v+1} y_{-k+1}, & \text { if } n=(k+1) v+2, \\
\vdots & \\
\left(\frac{\alpha_{4}}{\alpha_{5}}\right)^{v+1} y_{-1}, & \text { if } n=(k+1) v+k, \\
\left(\frac{\alpha_{4}}{\alpha_{5}}\right)^{v+1} y_{0}, & \text { if } n=(k+1) v+k+1,\end{cases} \\
& 0<z_{n} \leq \begin{cases}\left(\frac{\alpha_{7}}{\alpha_{8}}\right)^{v+1} z_{-k}, & \text { if } n=(k+1) v+1, \\
\left(\frac{\alpha_{7}}{\alpha_{8}}\right)^{v+1} z_{-k+1}, & \text { if } n=(k+1) v+2, \\
\vdots & \\
\left(\frac{\alpha_{7}}{\alpha_{8}}\right)^{v+1} z_{-1}, & \text { if } n=(k+1) v+k, \\
\left(\frac{\alpha_{7}}{\alpha_{8}}\right)^{v+1} z_{0}, & \text { if } n=(k+1) v+k+1 .\end{cases}
\end{aligned}
$$

Proof. For $v=0$, (31)-(33) are true trivially. Suppose that (31)-(33) are true for $v=\sigma \geq 1$, that is,

$$
\begin{aligned}
& 0<x_{n} \leq \begin{cases}\left(\frac{\alpha_{1}}{\alpha_{2}}\right)^{\sigma+1} x_{-k}, & \text { if } n=(k+1) \sigma+1, \\
\left(\frac{\alpha_{1}}{\alpha_{2}}\right)^{\sigma+1} x_{-k+1}, & \text { if } n=(k+1) \sigma+2, \\
\vdots & \text { if } n=(k+1) \sigma+k, \\
\left(\frac{\alpha_{1}}{\alpha_{2}}\right)^{\sigma+1} x_{-1}, & \\
\left(\frac{\alpha_{1}}{\alpha_{2}}\right)^{\sigma+1} x_{0}, & \text { if } n=(k+1) \sigma+(k+1),\end{cases} \\
& 0<y_{n} \leq \begin{cases}\left(\frac{\alpha_{4}}{\alpha_{5}}\right)^{\sigma+1} y_{-k}, & \text { if } n=(k+1) \sigma+1, \\
\left(\frac{\alpha_{4}}{\alpha_{5}}\right)^{\sigma+1} y_{-k+1}, & \text { if } n=(k+1) \sigma+2, \\
\vdots & \\
\left(\frac{\alpha_{4}}{\alpha_{5}}\right)^{\sigma+1} y_{-1}, & \text { if } n=(k+1) \sigma+k, \\
\left(\frac{\alpha_{4}}{\alpha_{5}}\right)^{\sigma+1} y_{0}, & \text { if } n=(k+1) \sigma+k+1,\end{cases} \\
& 0<z_{n} \leq \begin{cases}\left(\frac{\alpha_{7}}{\alpha_{8}}\right)^{\sigma+1} z_{-k}, & \text { if } n=(k+1) \sigma+1, \\
\left(\frac{\alpha_{7}}{\alpha_{8}}\right)^{\sigma+1} z_{-k+1}, & \text { if } n=(k+1) \sigma+1, \\
\vdots & \\
\left(\frac{\alpha_{7}}{\alpha_{8}}\right)^{\sigma+1} z_{-1}, & \text { if } n=(k+1) \sigma+k, \\
\left(\frac{\alpha_{7}}{\alpha_{8}}\right)^{\sigma+1} z_{0}, & \text { if } n=(k+1) \sigma+k+1 .\end{cases}
\end{aligned}
$$


Finally for $v=\sigma+1$ and using (7), one obtains

$$
\begin{aligned}
& x_{(k+1)(\sigma+1)+1}=\frac{\alpha_{1} x_{(k+1) \sigma+1}}{\alpha_{2}+\alpha_{3} \prod_{i=0}^{k} y_{(k+1) \sigma+k+1-i}} \leq \frac{\alpha_{1}}{\alpha_{2}} x_{(k+1) \sigma+1} \leq\left(\frac{\alpha_{1}}{\alpha_{2}}\right)^{\sigma+2} x_{-k}, \\
& x_{(k+1)(\sigma+1)+2}=\frac{\alpha_{1} x_{(k+1) \sigma+2}}{\alpha_{2}+\alpha_{3} \prod_{i=0}^{k} y_{(k+1) \sigma+k+2-i}} \leq \frac{\alpha_{1}}{\alpha_{2}} x_{(k+1) \sigma+2} \leq\left(\frac{\alpha_{1}}{\alpha_{2}}\right)^{\sigma+2} x_{-k+1} \text {, } \\
& \text { : } \\
& x_{(k+1)(\sigma+1)+k}=\frac{\alpha_{1} x_{(k+1) \sigma+k}}{\alpha_{2}+\alpha_{3} \prod_{i=0}^{k} y_{(k+1) \sigma+2 k-i}} \leq \frac{\alpha_{1}}{\alpha_{2}} x_{(k+1) \sigma+k} \leq\left(\frac{\alpha_{1}}{\alpha_{2}}\right)^{\sigma+2} x_{-1}, \\
& x_{(k+1)(\sigma+1)+k+1}=\frac{\alpha_{1} x_{(k+1) \sigma+k+1}}{\alpha_{2}+\alpha_{3} \prod_{i=0}^{k} y_{(k+1) \sigma+2 k+1-i}} \leq \frac{\alpha_{1}}{\alpha_{2}} x_{(k+1) \sigma+k+1} \leq\left(\frac{\alpha_{1}}{\alpha_{2}}\right)^{\sigma+2} x_{0} \text {, } \\
& y_{(k+1)(\sigma+1)+1}=\frac{\alpha_{4} y_{(k+1) \sigma+1}}{\alpha_{5}+\alpha_{6} \prod_{i=0}^{k} z_{(k+1) \sigma+k+1-i}} \leq \frac{\alpha_{4}}{\alpha_{5}} y_{(k+1) \sigma+1} \leq\left(\frac{\alpha_{4}}{\alpha_{5}}\right)^{\sigma+2} y_{-k}, \\
& y_{(k+1)(\sigma+1)+2}=\frac{\alpha_{4} y_{(k+1) \sigma+2}}{\alpha_{5}+\alpha_{6} \prod_{i=0}^{k} z_{(k+1) \sigma+k+2-i}} \leq \frac{\alpha_{4}}{\alpha_{5}} y_{(k+1) \sigma+2} \leq\left(\frac{\alpha_{4}}{\alpha_{5}}\right)^{\sigma+2} y_{-k+1} \text {, } \\
& \text { : } \\
& y_{(k+1)(\sigma+1)+k}=\frac{\alpha_{4} y_{(k+1) \sigma+k}}{\alpha_{5}+\alpha_{6} \prod_{i=0}^{k} z_{(k+1) \sigma+2 k-i}} \leq \frac{\alpha_{4}}{\alpha_{5}} y_{(k+1) \sigma+k} \leq\left(\frac{\alpha_{4}}{\alpha_{5}}\right)^{\sigma+2} y_{-1} \text {, } \\
& y_{(k+1)(\sigma+1)+k+1}=\frac{\alpha_{4} y_{(k+1) \sigma+k+1}}{\alpha_{5}+\alpha_{6} \prod_{i=0}^{k} z_{(k+1) \sigma+2 k+1-i}} \leq \frac{\alpha_{4}}{\alpha_{5}} y_{(k+1) \sigma+k+1} \leq\left(\frac{\alpha_{4}}{\alpha_{5}}\right)^{\sigma+2} y_{0} \text {, } \\
& z_{(k+1)(\sigma+1)+1}=\frac{\alpha_{7} z_{(k+1) \sigma+1}}{\alpha_{8}+\alpha_{9} \prod_{i=0}^{k} x_{(k+1) \sigma+k+1-i}} \leq \frac{\alpha_{7}}{\alpha_{8}} z_{(k+1) \sigma+1} \leq\left(\frac{\alpha_{7}}{\alpha_{8}}\right)^{\sigma+2} z_{-k} \\
& z_{(k+1)(\sigma+1)+2}=\frac{\alpha_{7} z_{(k+1) \sigma+2}}{\alpha_{8}+\alpha_{9} \prod_{i=0}^{k} x_{(k+1) \sigma+k+2-i}} \leq \frac{\alpha_{7}}{\alpha_{8}} z_{(k+1) \sigma+2} \leq\left(\frac{\alpha_{7}}{\alpha_{8}}\right)^{\sigma+2} z_{-k+1} \text {, } \\
& \text { : } \\
& z_{(k+1)(\sigma+1)+k}=\frac{\alpha_{7} z_{(k+1) \sigma+k}}{\alpha_{8}+\alpha_{9} \prod_{i=0}^{k} x_{(k+1) \sigma+2 k-i}} \leq \frac{\alpha_{7}}{\alpha_{8}} z_{(k+1) \sigma+k} \leq\left(\frac{\alpha_{7}}{\alpha_{8}}\right)^{\sigma+2} z_{-1}, \\
& z_{(k+1)(\sigma+1)+k+1}=\frac{\alpha_{7} z_{(k+1) \sigma+k+1}}{\alpha_{8}+\alpha_{9} \prod_{i=0}^{k} x_{(k+1) \sigma+2 k+1-i}} \leq \frac{\alpha_{7}}{\alpha_{8}} z_{(k+1) \sigma+k+1} \leq\left(\frac{\alpha_{7}}{\alpha_{8}}\right)^{\sigma+2} z_{0} \text {. }
\end{aligned}
$$

From (37)-(39), one has required result.

Lemma 2. If (11) holds, then $\left\{\Omega_{n}\right\}_{n=-k}^{\infty}$ of (7) is bounded.

Proof. It is consequence of Theorem 7.

Remark 1. From above theoretical studies, we conclude that no equilibria are locally stable except $P_{000}$, and hence in the following section we will investigate that it is globally asymptotically stable under certain parametric conditions.

\section{Global Dynamics about $P_{000}$}

Theorem 8. $P_{000}$ of system (7) is globally asymptotically stable if conditions, which are depicted in (11), hold.

Proof. By Theorem 1, $P_{000}$ of (7) is a sink and also $\left\{\Omega_{n}\right\}_{n=-k}^{\infty}$ is bounded by Lemma 2 . In order to get the required statement, it is adequate to prove $\left\{\left(x_{n}, y_{n}, z_{n}\right)\right\}$ is nonincreasing. In view of (11), from (7), one gets $x_{n+1}<x_{n-k}$, which again gives $x_{(k+1) n+1}<x_{(k+1) n-k}$ and $x_{(k+1) n+k+2}<x_{(k+1) n+1}$. Thus, $\left\{\left(x_{n}\right)\right\}$ is decreasing because $\left\{x_{(k+1) n+1}\right\},\left\{x_{(k+1) n+2}\right\}, \ldots$, 
$\left\{x_{(k+1) n+k+1}\right\}$ are nonincreasing. Similarly, it is easy to investigate that $\left\{\left(y_{n}\right)\right\}$ and $\left\{\left(z_{n}\right)\right\}$ are also decreasing. This implies that $\lim _{n \longrightarrow \infty}\left(x_{n}, y_{n}, z_{n}\right)=P_{000}$.

Lemma 3. If

$$
\frac{\alpha_{1}}{\alpha_{2}}>1, \frac{\alpha_{4}}{\alpha_{5}}>1, \frac{\alpha_{7}}{\alpha_{8}}>1,
$$

then for $\left\{\Omega_{n}\right\}_{n=-k}^{\infty}$ of (7), the following holds:

$$
\begin{aligned}
& \text { (i) } x_{n} \longrightarrow \infty \text { if } y_{n} \longrightarrow 0 \text {. } \\
& \text { (ii) } y_{n} \longrightarrow \infty \text { if } z_{n} \longrightarrow 0 \text {. } \\
& \text { (iii) } z_{n} \longrightarrow \infty \text { if } x_{n} \longrightarrow 0 \text {. }
\end{aligned}
$$

Lemma 4. If (38) holds, then for $(\ell=k,-k+1, \ldots, 1,0)$, one gets the following invariant intervals:

(i) $\left(x_{-\ell}, y_{-\ell}, z_{-\ell}\right) \in\left(0, \sqrt{[k+1]}\left(\alpha_{7}-\alpha_{8} / \alpha_{9}\right)\right) \times$

$\left(0, \sqrt{[k+1]} \quad\left(\alpha_{1}-\alpha_{2} / \alpha_{3}\right)\right) \times\left(\sqrt{[k+1]}\left(\alpha_{4}-\alpha_{5} / \alpha_{6}\right), \infty\right)$

$\Rightarrow\left(x_{n}, y_{n}, z_{n}\right) \in\left(0, \sqrt{[k+1]}\left(\alpha_{7}-\alpha_{8} / \alpha_{9}\right)\right) \times(0, \sqrt{[k+1]}$

$\left.\left(\alpha_{1}-\alpha_{2} / \alpha_{3}\right)\right) \times\left(\sqrt{[k+1]}\left(\alpha_{4}-\alpha_{5} / \alpha_{6}\right), \infty\right), \forall n \geq 1$.

(ii) $\left(x_{-\ell}, y_{-\ell}, z_{-\ell}\right) \in\left(\sqrt{[k+1]}\left(\alpha_{7}-\alpha_{8} / \alpha_{9}\right), \infty\right) \times$ $\left(\sqrt{[k+1]}\left(\alpha_{1}-\alpha_{2} / \quad \alpha_{3}\right), \infty\right) \times\left(0, \sqrt{[k+1]}\left(\alpha_{4}-\right.\right.$ $\left.\left.\alpha_{5} / \alpha_{6}\right)\right) \Rightarrow\left(x_{n}, y_{n}, z_{n}\right) \in\left(\sqrt{[k+1]}\left(\alpha_{7}-\alpha_{8} / \alpha_{9}\right), \infty\right)$ $\times\left(\sqrt{[k+1]}\left(\alpha_{1}-\alpha_{2} / \alpha_{3}\right), \infty\right) \times\left(0, \sqrt{[k+1]} \quad\left(\alpha_{4}-\right.\right.$ $\left.\left.\alpha_{5} / \alpha_{6}\right)\right), \forall n \geq 1$.

Proof. This follows by induction.

\section{Convergence Rate}

Theorem 9. If (11) is true, then error vector

$$
\varrho_{n}=\left(\begin{array}{c}
\varrho_{n}^{1} \\
\vdots \\
\varrho_{n-k}^{1} \\
\varrho_{n}^{2} \\
\vdots \\
\varrho_{n-k}^{2} \\
\varrho_{n}^{3} \\
\vdots \\
\varrho_{n-k}^{3}
\end{array}\right),
$$

of positive solution $\left\{\Omega_{n}\right\}_{n=-k}^{\infty}$ of (7) satisfies the following relations:

$$
\begin{gathered}
\lim _{n \rightarrow \infty} \sqrt{[n]}\left\|\varrho_{n}\right\|=|\nu J|_{P_{000}} \mid, \\
\lim _{n \longrightarrow \infty} \frac{\left\|\varrho_{n+1}\right\|}{\left\|\varrho_{n}\right\|}=|\nu J|_{P_{000}} \mid,
\end{gathered}
$$

where $|\nu J|_{P_{000}} \mid$ is equivalent to modulus of one of the characteristic roots of $\left.J\right|_{P_{000}}$ calculated at $P_{000}$.

Proof. If $\left\{\Omega_{n}\right\}_{n=-k}^{\infty}$ is an arbitrary solution of (7) such that $\lim _{n \longrightarrow \infty} x_{n}=x, \lim _{n \longrightarrow \infty} y_{n}=y$ and $\lim _{n \longrightarrow \infty} z_{n}=z$, then

$$
\begin{aligned}
x_{n+1}-x= & \frac{\alpha_{1} x_{n-k}}{\alpha_{2}+\alpha_{3} \prod_{i=0}^{k} y_{n-i}}-\frac{\alpha_{1} x}{\alpha_{2}+\alpha_{3} y^{k+1}} \\
= & \frac{\alpha_{1}}{\alpha_{2}+\alpha_{3} \prod_{i=0}^{k} y_{n-i}}\left(x_{n-k}-x\right)-\frac{\alpha_{1} \alpha_{3} x \prod_{i=1}^{k} y_{n-i}}{\left(\alpha_{2}+\alpha_{3} \prod_{i=0}^{k} y_{n-i}\right)\left(\alpha_{2}+\alpha_{3} y^{k+1}\right)}\left(y_{n}-y\right) \\
& -\frac{\alpha_{1} \alpha_{3} x y \prod_{i=2}^{k} y_{n-i}}{\left(\alpha_{2}+\alpha_{3} \prod_{i=0}^{k} y_{n-i}\right)\left(\alpha_{2}+\alpha_{3} y^{k+1}\right)}\left(y_{n-1}-y\right)-\ldots \\
& -\frac{\alpha_{1} \alpha_{3} x y^{k-1} y_{n-k}}{\left(\alpha_{2}+\alpha_{3} \prod_{i=0}^{k} y_{n-i}\right)\left(\alpha_{2}+\alpha_{3} y^{k+1}\right)}\left(y_{n-k+1}-y\right) \\
& -\frac{\alpha_{1} \alpha_{3} x y^{k}}{\left(\alpha_{2}+\alpha_{3} \prod_{i=0}^{k} y_{n-i}\right)\left(\alpha_{2}+\alpha_{3} y^{k+1}\right)}\left(y_{n-k}-y\right) .
\end{aligned}
$$

Similarly from (7), we obtain 


$$
\begin{aligned}
& y_{n+1}-y=\frac{\alpha_{4}}{\alpha_{5}+\alpha_{6} \prod_{i=0}^{k} z_{n-i}}\left(y_{n-k}-y\right) \\
& -\frac{\alpha_{4} \alpha_{6} y \prod_{i=1}^{k} z_{n-i}}{\left(\alpha_{5}+\alpha_{6} \prod_{i=0}^{k} z_{n-i}\right)\left(\alpha_{5}+\alpha_{6} z^{k+1}\right)}\left(z_{n}-z\right) \\
& -\frac{\alpha_{4} \alpha_{6} y z \prod_{i=2}^{k} z_{n-i}}{\left(\alpha_{5}+\alpha_{6} \prod_{i=0}^{k} z_{n-i}\right)\left(\alpha_{5}+\alpha_{6} z^{k+1}\right)}\left(z_{n-1}-z\right)-\cdots \\
& -\frac{\alpha_{4} \alpha_{6} y z^{k-1} z_{n-k}}{\left(\alpha_{5}+\alpha_{6} \prod_{i=0}^{k} z_{n-i}\right)\left(\alpha_{5}+\alpha_{6} z^{k+1}\right)}\left(z_{n-k+1}-z\right) \\
& -\frac{\alpha_{4} \alpha_{6} y z^{k}}{\left(\alpha_{5}+\alpha_{6} \prod_{i=0}^{k} z_{n-i}\right)\left(\alpha_{5}+\alpha_{6} z^{k+1}\right)}\left(z_{n-k}-z\right), \\
& z_{n+1}-z=-\frac{\alpha_{7} \alpha_{9} z \prod_{i=1}^{k} x_{n-i}}{\left(\alpha_{8}+\alpha_{9} \prod_{i=0}^{k} x_{n-i}\right)\left(\alpha_{8}+\alpha_{9} z^{k+1}\right)}\left(x_{n}-x\right) \\
& -\frac{\alpha_{7} \alpha_{9} z x \prod_{i=2}^{k} x_{n-i}}{\left(\alpha_{8}+\alpha_{9} \prod_{i=0}^{k} x_{n-i}\right)\left(\alpha_{8}+\alpha_{9} x^{k+1}\right)}\left(x_{n-1}-x\right)-\cdots \\
& -\frac{\alpha_{7} \alpha_{9} z x^{k-1} z_{n-k}}{\left(\alpha_{8}+\alpha_{9} \prod_{i=0}^{k} x_{n-i}\right)\left(\alpha_{8}+\alpha_{9} x^{k+1}\right)}\left(x_{n-k+1}-x\right) \\
& -\frac{\alpha_{7} \alpha_{9} z x^{k}}{\left(\alpha_{8}+\alpha_{9} \prod_{i=0}^{k} x_{n-i}\right)\left(\alpha_{8}+\alpha_{9} x^{k+1}\right)}\left(x_{n-k}-x\right) \\
& +\frac{\alpha_{7}}{\alpha_{8}+\alpha_{9} \prod_{i=0}^{k} x_{n-i}}\left(z_{n-k}-z\right) .
\end{aligned}
$$

Now, set

$$
\begin{aligned}
& \varrho_{n}^{1}=x_{n}-x, \\
& \varrho_{n}^{2}=y_{n}-y, \\
& \varrho_{n}^{3}=z_{n}-z .
\end{aligned}
$$

Utilizing (43) in (41) and (42), we get

$$
\begin{aligned}
\varrho_{n+1}^{1}= & A_{n}^{n-k} \varrho_{n-k}^{1}+B_{n}^{n} \varrho_{n}^{2}+B_{n}^{n-1} \varrho_{n-1}^{2}+\cdots+B_{n}^{n-k+1} \varrho_{n-k+1}^{2} \\
& +B_{n}^{n-k} \varrho_{n-k}^{2}, \\
\varrho_{n+1}^{2}= & C_{n}^{n-k} \varrho_{n-k}^{2}+D_{n}^{n} \varrho_{n}^{3}+D_{n}^{n-1} \varrho_{n-1}^{3}+\cdots+D_{n}^{n-k+1} \varrho_{n-k+1}^{3} \\
& +D_{n}^{n-k} \varrho_{n-k}^{3}, \\
\varrho_{n+1}^{3}= & E_{n}^{n} \varrho_{n}^{1}+E_{n}^{n-1} \varrho_{n-1}^{1}+\cdots+E_{n}^{n-k+1} \varrho_{n-k+1}^{1}+E_{n}^{n-k} \varrho_{n-k}^{1} \\
& +F_{n}^{n-k} \varrho_{n-k}^{3},
\end{aligned}
$$

where

$$
\begin{aligned}
& A_{n}^{n-k}=\frac{\alpha_{1}}{\alpha_{2}+\alpha_{3} \prod_{i=0}^{k} y_{n-i}}, \\
& B_{n}^{n}=-\frac{\alpha_{1} \alpha_{3} x \prod_{i=1}^{k} y_{n-i}}{\left(\alpha_{2}+\alpha_{3} \prod_{i=0}^{k} y_{n-i}\right)\left(\alpha_{2}+\alpha_{3} y^{k+1}\right)}, \\
& B_{n}^{n-1}=-\frac{\alpha_{1} \alpha_{3} x y \prod_{i=2}^{k} y_{n-i}}{\left(\alpha_{2}+\alpha_{3} \prod_{i=0}^{k} y_{n-i}\right)\left(\alpha_{2}+\alpha_{3} y^{k+1}\right)}, \\
& \vdots \\
& B_{n}^{n-k+1}=-\frac{\alpha_{1} \alpha_{3} x y^{k-1} y_{n-k}}{\left(\alpha_{2}+\alpha_{3} \prod_{i=0}^{k} y_{n-i}\right)\left(\alpha_{2}+\alpha_{3} y^{k+1}\right)}, \\
& B_{n}^{n-k}=-\frac{\alpha_{1} \alpha_{3} x y^{k}}{\left(\alpha_{2}+\alpha_{3} \prod_{i=0}^{k} y_{n-i}\right)\left(\alpha_{2}+\alpha_{3} y^{k+1}\right)}, \\
& C_{n}^{n-k}=\frac{\alpha_{4}}{\alpha_{5}+\alpha_{6} \prod_{i=0}^{k} z_{n-i}}, \\
& D_{n}^{n}=-\frac{\alpha_{4} \alpha_{6} y \prod_{i=1}^{k} z_{n-i}}{\left(\alpha_{5}+\alpha_{6} \prod_{i=0}^{k} z_{n-i}\right)\left(\alpha_{5}+\alpha_{6} z^{k+1}\right)}, \\
& D_{n}^{n-1}=-\frac{\alpha_{4} \alpha_{6} y z \prod_{i=2}^{k} z_{n-i}}{\left(\alpha_{5}+\alpha_{6} \prod_{i=0}^{k} z_{n-i}\right)\left(\alpha_{5}+\alpha_{6} z^{k+1}\right)}, \\
& \text { : } \\
& D_{n}^{n-k+1}=-\frac{\alpha_{4} \alpha_{6} y z^{k-1} z_{n-k}}{\left(\alpha_{5}+\alpha_{6} \prod_{i=0}^{k} z_{n-i}\right)\left(\alpha_{5}+\alpha_{6} z^{k+1}\right)}, \\
& D_{n}^{n-k}=-\frac{\alpha_{4} \alpha_{6} y z^{k}}{\left(\alpha_{5}+\alpha_{6} \prod_{i=0}^{k} z_{n-i}\right)\left(\alpha_{5}+\alpha_{6} z^{k+1}\right)} \text {, } \\
& E_{n}^{n}=-\frac{\alpha_{7} \alpha_{9} z \prod_{i=1}^{k} x_{n-i}}{\left(\alpha_{8}+\alpha_{9} \prod_{i=0}^{k} x_{n-i}\right)\left(\alpha_{8}+\alpha_{9} z^{k+1}\right)}, \\
& E_{n}^{n-1}=-\frac{\alpha_{7} \alpha_{9} z x \prod_{i=2}^{k} x_{n-i}}{\left(\alpha_{8}+\alpha_{9} \prod_{i=0}^{k} x_{n-i}\right)\left(\alpha_{8}+\alpha_{9} x^{k+1}\right)}, \\
& \text { : } \\
& E_{n}^{n-k+1}=-\frac{\alpha_{7} \alpha_{9} z x^{k-1} z_{n-k}}{\left(\alpha_{8}+\alpha_{9} \prod_{i=0}^{k} x_{n-i}\right)\left(\alpha_{8}+\alpha_{9} x^{k+1}\right)}, \\
& E_{n}^{n-k}=-\frac{\alpha_{7} \alpha_{9} z x^{k}}{\left(\alpha_{8}+\alpha_{9} \prod_{i=0}^{k} x_{n-i}\right)\left(\alpha_{8}+\alpha_{9} x^{k+1}\right)}, \\
& F_{n}^{n-k}=\frac{\alpha_{7}}{\alpha_{8}+\alpha_{9} \prod_{i=0}^{k} x_{n-i}} .
\end{aligned}
$$

From (45) and (46), we get 


$$
\begin{aligned}
& \lim _{n \longrightarrow \infty} A_{n}^{n-k}=\frac{\alpha_{1}}{\alpha_{2}+\alpha_{3} y^{k+1}}, \\
& \lim _{n \longrightarrow \infty} B_{n}^{n}=\lim _{n \longrightarrow \infty} B_{n}^{n-1}=\cdots=\lim _{n \longrightarrow \infty} B_{n}^{n-k+1}=\lim _{n \longrightarrow \infty} B_{n}^{n-k}=-\frac{\alpha_{1} \alpha_{3} x y^{k}}{\left(\alpha_{2}+\alpha_{3} y^{k+1}\right)^{2}}, \\
& \lim _{n \longrightarrow \infty} C_{n}^{n-k}=\frac{\alpha_{4}}{\alpha_{5}+\alpha_{6} z^{k+1}}, \\
& \lim _{n \longrightarrow \infty} D_{n}^{n}=\lim _{n \longrightarrow \infty} D_{n}^{n-1}=\cdots=\lim _{n \longrightarrow \infty} D_{n}^{n-k+1}=\lim _{n \longrightarrow \infty} D_{n}^{n-k}=-\frac{\alpha_{4} \alpha_{6} y z^{k}}{\left(\alpha_{5}+\alpha_{6} z^{k+1}\right)^{2}}, \\
& \lim _{n \longrightarrow \infty} E_{n}^{n}=\lim _{n \longrightarrow \infty} E_{n}^{n-1}=\cdots=\lim _{n \longrightarrow \infty} E_{n}^{n-k+1}=\lim _{n \longrightarrow \infty} E_{n}^{n-k}=-\frac{\alpha_{7} \alpha_{9} x^{k} z}{\left(\alpha_{8}+\alpha_{9} x^{k+1}\right)^{2}}, \\
& \lim _{n \longrightarrow \infty} F_{n}^{n-k}=\frac{\alpha_{7}}{\alpha_{8}+\alpha_{9} x^{k+1}} .
\end{aligned}
$$

So, we have the limiting system [26]:

$$
\varrho_{n+1}=K \varrho_{n},
$$

where $\varrho_{n}$ is depicted in (39) and $K$ is same as $\left.J\right|_{\wedge}$ about $\wedge$. In particular, about $P_{000}$ it becomes

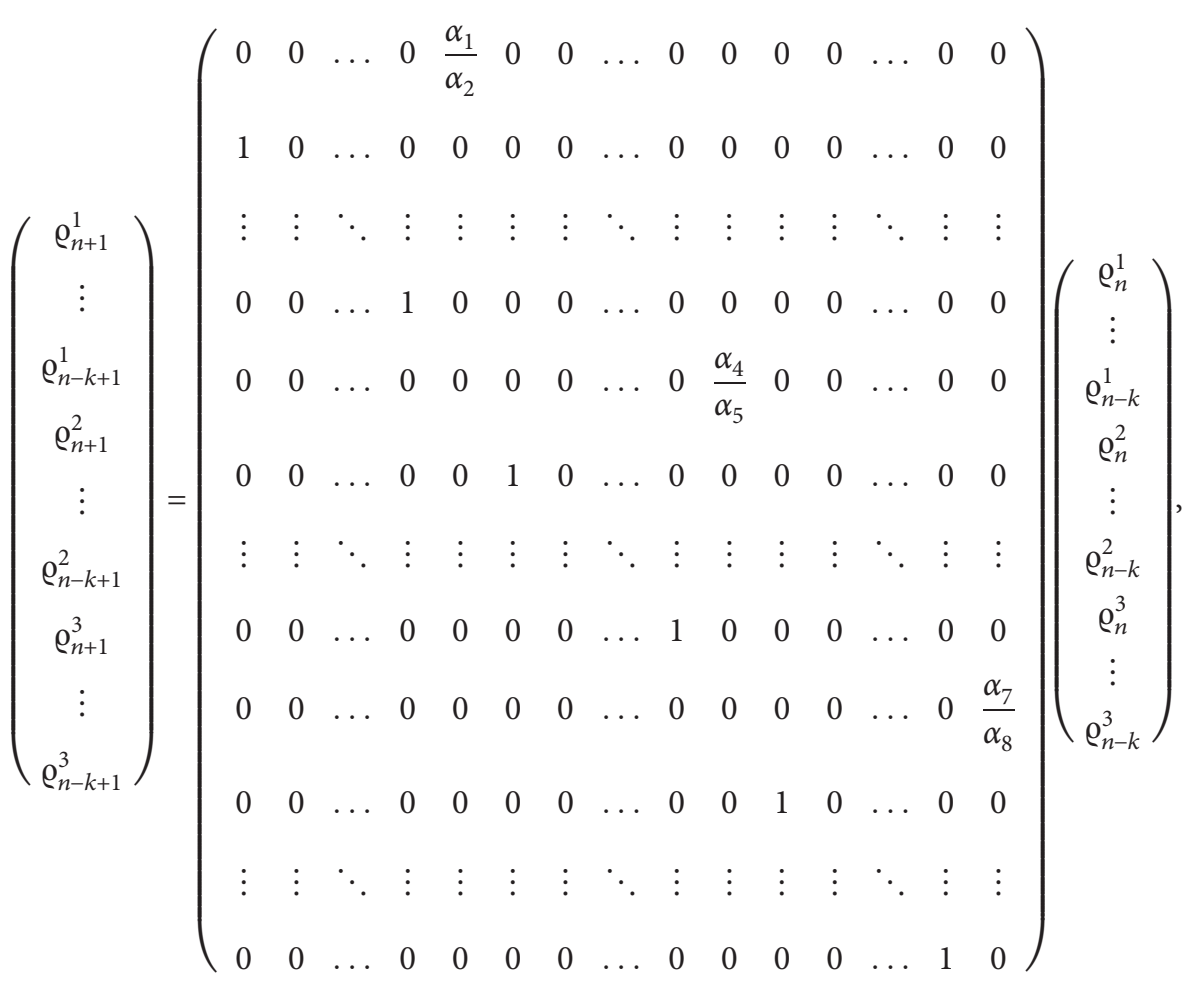

which is same as linearized system of (7) about $P_{000}$.

\section{Numerical Simulations}

We will present numerous numerical examples, which represent different dynamical behaviors of system (7), in this section.
Example 1. Figure 1 indicates the dynamics of (7) about $P_{000}$ if $k=2, \quad \alpha_{\ell}(\ell=1,2, \ldots, 9)$ respectively, are $14,15,3,6,5,1,11,13$, and 3 and initial values $x_{-\ell}, \ell_{-\ell}$, $z_{-\ell}(\ell=2,1,0)$, respectively, are $0.07,0.9,0.02,0.9,0.9,1.4$, $4.9,0.9$, and 0.8 . More precisely, Figures $1(\mathrm{a})-1(\mathrm{c})$ show that $P_{000}$ of $(7)$ is a sink while its corresponding global attractor is shown is Figure 1(d). Figure 1(d) clearly indicates that all 


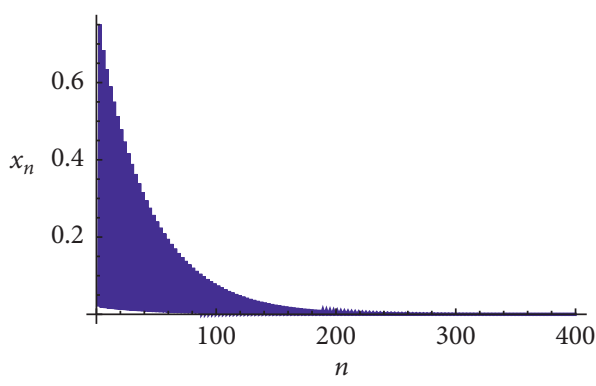

(a)

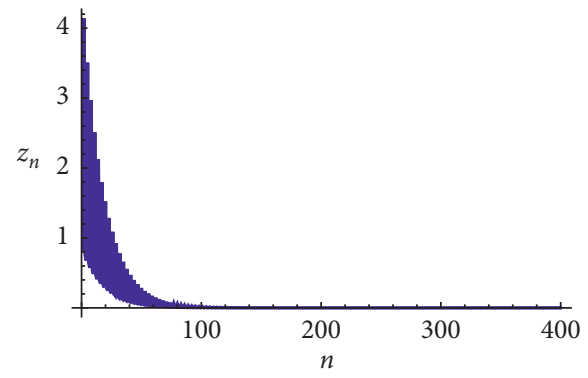

(c)

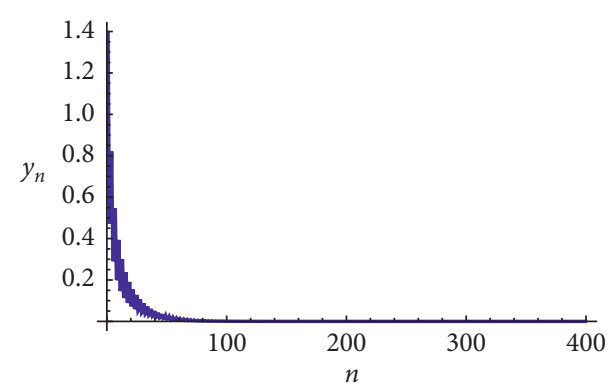

(b)

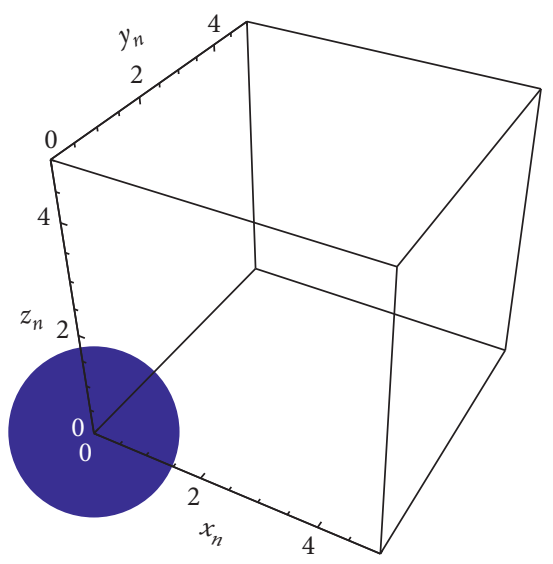

(d)

Figure 1: Dynamics of (7).

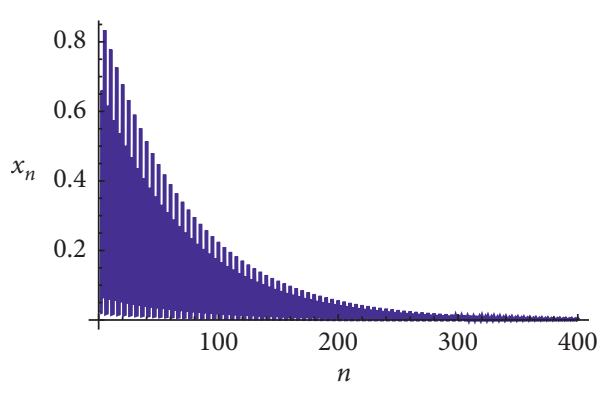

(a)

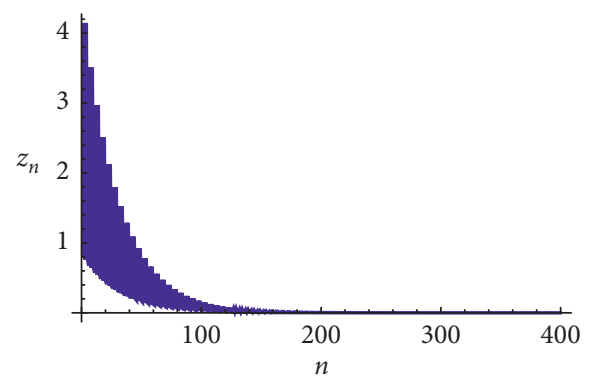

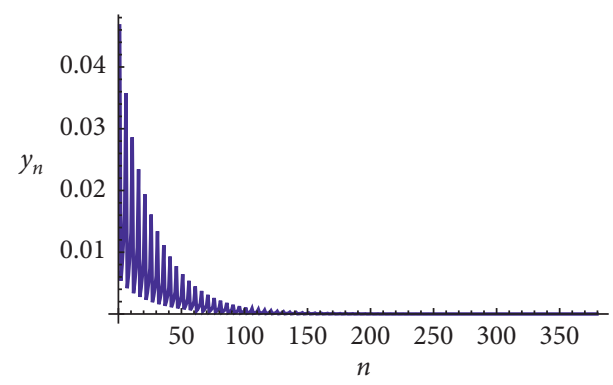

(b)

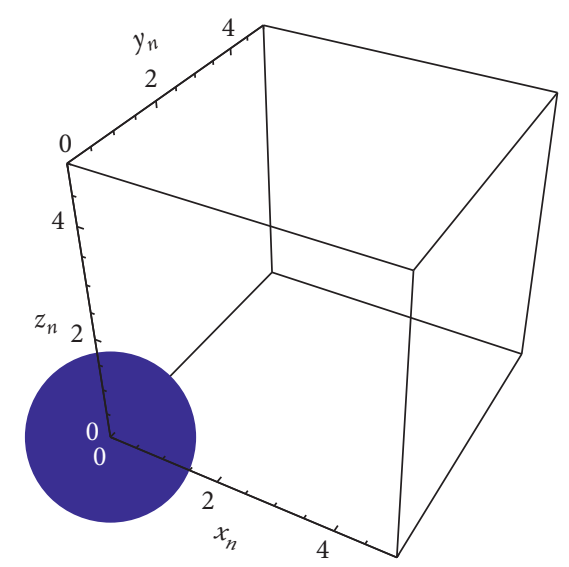

(d)

(c)

Figure 2: Dynamics of (7). 


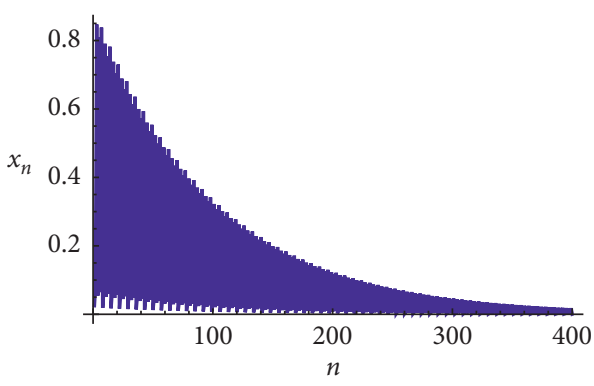

(a)

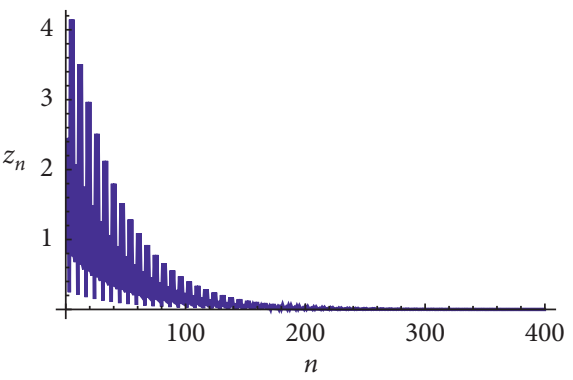

(c)

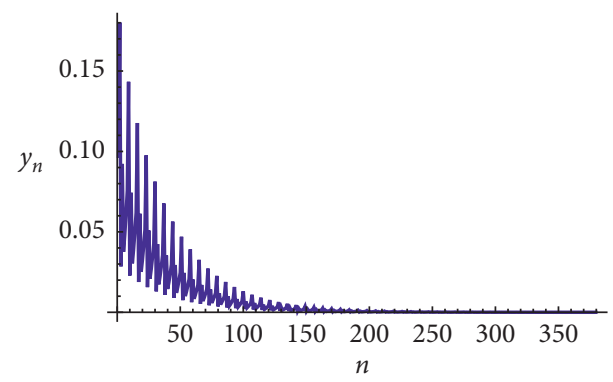

(b)

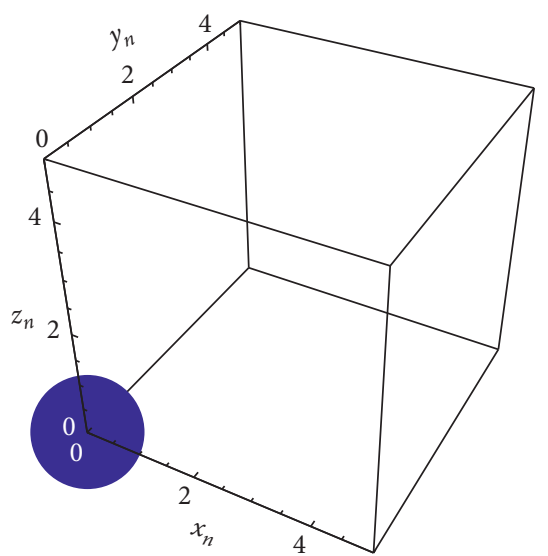

(d)

Figure 3: Dynamics of (7).

solutions go towards $P_{000}$; this means that $P_{000}$ is globally asymptotically stable. Hence, this simulation agrees with the obtained results.

Example 2. Figure 2 indicates the same dynamics of (7) about $P_{000}$ if $k=4, \alpha_{\ell}(\ell=1,2, \ldots, 9)$, respectively, are 14 , $15,33,6,5,2,11,13$, and 3 and initial values $x_{-\ell}, \ell_{-\ell}$, $z_{-\ell}(\ell=4,3,2,1,0)$, respectively, are $0.07,0.9,0.07,0.9,0.02$, $0.9,0.9,0.9,0.9,1.4,4.9,1.4,4.9,0.9$, and 0.8 .

Example 3. Figure 3 indicates the dynamics of (7) about $P_{000}$ if $k=6, \alpha_{\ell}(\ell=1,2, \ldots, 9)$, respectively, are $14,15,33,6,5,2$, 11, 13, and 3 and initial values $x_{-\ell}, \ell_{-\ell}$, $z_{-\ell}(\ell=6,5,4,3,2,1,0)$, respectively, are $0.7,1.9,0.07,0.9$, $0.07,0.9,0.02,1.2,2.9,0.9,1.4,2.9,0.29,4.9,1.4,4.9,0.9$, and 0.8 .

\section{Concluding Remarks}

The presented work is a natural extension of [21-25]. In this work, we have investigated the global dynamics of higherorder rational system (7) which is our main contribution to dynamical system. We explored that system (7) has eight equilibria $P_{000}, P_{x 0 z}, P_{x y 0}, P_{0 y z}, P_{x 00}, P_{00 z}, P_{0 y 0}$, and $P_{x y z}$ under certain conditions on parameters $\alpha_{\ell}(\ell=1,2, \ldots, 9)$. We investigated that only $P_{000}$ is locally stable under the condition, which is depicted in (11), while the rest of the equilibria $P_{x 0 z}, P_{x y 0}, P_{0 y z}, P_{x 00}, P_{00 z}, P_{0 y 0}$, and $P_{x y z}$ are locally unstable. Further, we have studied the global dynamics, boundedness, and rate of convergence of positive solution of (7). Finally, we have verified theoretical results numerically [2].

\section{Data Availability}

All the data utilized in this article have been included, and the sources from where they were adopted were cited accordingly.

\section{Conflicts of Interest}

The authors declare that they have no conflicts of interest regarding the publication of this paper.

\section{Acknowledgments}

This study was partially supported by the Higher Education Commission of Pakistan.

\section{References}

[1] H. Sedaghat, Nonlinear Difference Equations: Theory with Applications to Social Science Models, Kluwer Academic, Dordrecht, Netherlands, 2003.

[2] V. L. Kocic and G. Ladas, Global Behavior of Nonlinear Difference Equations of Higher-Order with Applications, Kluwer Academic, Dordrecht, Netherlands, 1993.

[3] E. A. Grove and G. Ladas, Periodicities in Nonlinear Difference Equations, Chapman and Hall/CRC, Boca Raton, FL, USA, 2004. 
[4] M. R. S. Kulenović and G. Ladas, Dynamics of Second-Order Rational Difference Equations, Chapman and Hall/CRC, London, UK, 2002.

[5] E. Camouzis and G. Ladas, Dynamics of Third-Order Rational Difference Equations: With Open Problems and Conjectures, Chapman and Hall/HRC, Boca Raton, FL, USA, 2007.

[6] A. E. Hamza and R. Khalaf-All, "Global behavior of a higher order difference equation," Journal of Mathematics and Statistics, vol. 3, no. 1, pp. 17-20, 2007.

[7] R. Khalaf-Allah, "Asymptotic behavior and periodic nature of two difference Equations," Ukrainian Mathematical Journal, vol. 61, no. 6, pp. 834-838, 2009.

[8] V. L. Kocic and G. Ladas, "Global attractivity in a secondorder nonlinear difference equation," Journal of Mathematical Analysis and Applications, vol. 180, no. 1, pp. 144-150, 1993.

[9] M. R. S. Kulenović, G. Ladas, and N. P. Prokup, "A rational difference equation," Computers \& Mathematics with Applications, vol. 41, pp. 671-678, 2001.

[10] Q. Zhang, W. Zhang, Y. Shao, and J. Liu, "On the system of high order rational difference equations," International Scholarly Research Notices, vol. 2014, Article ID 760502, 5 pages, 2014

[11] S. Kalabusisić, M. R. S. Kulenović, and E. Pilav, "Global dynamics of a competitive system of rational difference equations in the plane," Advances in Difference Equations, vol. 2009, Article ID 132802, 30 pages, 2009.

[12] S. Kalabusisić, M. R. S. Kulenović, and E. Pilav, "Dynamics of a two-dimensional system of rational difference equations of Leslie-Gower type," Advances in Difference Equations, 2011.

[13] S. Kalabusisić, M. R. S. Kulenović, and E. Pilav, "multiple attractors for a competitive system of rational difference equations in the plane," Abstract and Applied Analysis, vol. 2011, Article ID 295308, 35 pages, 2011.

[14] M. Garić-Demirović, M. R. S. Kulenović, and M. Nurkanović, "Global behavior of four competitive rational systems of difference equations in the plane," Discrete Dynamics in Nature and Society, vol. 2009, Article ID 153058, 34 pages, 2009.

[15] E. M. Elsayed, "Solutions of rational difference systems of order two," Mathematical and Computer Modelling, vol. 55, no. 3-4, pp. 378-384, 2012.

[16] E. M. Elsayed, "Behavior and expression of the solutions of some rational difference equations," Journal of Computational Analysis and Applications, vol. 15, no. 1, pp. 73-81, 2013.

[17] A. Q. Khan and M. N. Qureshi, "Global dynamics of a competitive system of rational difference equations," Mathematical Methods in the Applied Sciences, vol. 38, no. 18, pp. 4786-4796, 2015.

[18] R. DeVault, G. Ladas, and S. W. Schultz, "On the recursive sequence $x_{n+1}=\left(A / x_{n}\right)+\left(1 / x_{n-2}\right)$." Proceedings of the American Mathematical Society, vol. 126, no. 11, pp. 32573262, 1998.

[19] R. M. Abu-Saris and R. DeVault, "Global stability of $y_{n}+1=A+y_{n} y_{n}-k$," Applied Mathematics Letters, vol. 16, no. 2, pp. 173-178, 2003.

[20] C. H. Gibbons, M. R. S. Kulenović, and G. Ladas, "On the recurrance sequence $x_{n+1}=\left(\alpha+\beta x_{n-1} / \gamma+x_{n}\right)$." Mathematical Sciences Research Hot-Line, vol. 4, no. 2, pp. 1-11, 2000.

[21] C. Çinar, "On the positive solutions of the difference equation $x_{n}+1=\left(x_{n}-1\right) /\left(1+x_{n} x_{n}-1\right), "$ Applied Mathematics and Computation, vol. 150, no. 1, pp. 21-24, 2004.

[22] M. Shojaei, R. Saadati, and H. Adibi, "Stability and periodic character of a rational third order difference equation," Chaos, Solitons \& Fractals, vol. 39, no. 3, pp. 1203-1209, 2009.
[23] I. Bajo and E. Liz, "Global behaviour of a second-order nonlinear difference equation," Journal of Difference Equations and Applications, vol. 17, no. 10, pp. 1471-1486, 2011.

[24] Q. Zhang, L. Yang, and J. Liu, "Dynamics of a system of rational third order difference equation," Advances in Difference Equations, vol. 2012, no. 1, 2012.

[25] S. M. Qureshi and A. Q. Khan, "Global dynamics of a $3 \times 6$ system of difference equations," Discrete Dynamics in Nature and Society, vol. 2019, Article ID 9797242, 14 pages, 2019.

[26] M. Pituk, "More on poincaré's and perron's Theorems for difference equations," Journal of Difference Equations and Applications, vol. 8, no. 3, pp. 201-216, 2002. 